\title{
Concepção, Implementação e Avaliação de um Agente Conversacional com Suporte à Aprendizagem Ubíqua
}

\author{
Leo Natan Paschoal, ICMC - USP, leonatanpaschoal@gmail.com \\ Patricia M. Mozzaquatro Chicon, NEAD - UNICRUZ, patriciamozzaquatro@gmail.com \\ Gilse Antoninha Morgental Falkembach, CINTED - UFRGS, gilsemf@gmail.com
}

Resumo. A ampla incorporação das tecnologias digitais na sociedade vem fazendo as ferramentas instrucionais adquirirem novos recursos, com a finalidade de atender as reais necessidades de seus utilizadores. Essa premissa é refletida nos Ambientes Virtuais de Aprendizagem, que vêm recebendo constantes atualizações, em direção aos sistemas ubíquos. Nessa perspectiva, a presente pesquisa se insere com o intuito de apresentar a concepção, implementação e avaliação de um agente conversacional, integrado ao Moodle, que auxilia estudantes de Engenharia de Software. Para tanto, o agente, denominado Ubibot, adapta suas mensagens de auxílio, considerando o contexto do estudante, a fim de oferecer acesso propício à aprendizagem ubíqua.

Palavras-Chave: Agentes Inteligentes. Aprendizagem Ubíqua. Computação Ciente de Contexto.

\section{Conception, Implementation and Evaluation of a Conversational Agent with Support for Ubiquitous Learning}

\begin{abstract}
The wide incorporation of digital technologies in society has made the instructional tools acquire new resources, in order to meet the real needs of its users. This premise is reflected in the virtual learning environments, which have been receiving constant updates, towards the ubiquitous systems. In this perspective, the present research is inserted with the intention of presenting the conception, implementation and evaluation of a conversational agent, integrated to Moodle, that helps students of Software Engineering. To this end, the agent, called Ubibot, adapts its help messages, considering the context of the student, in order to offer propitious access to ubiquitous learning.
\end{abstract}

Keywords: Intelligent Agent. Ubiquitous Learning. Context-Aware Computing.

\section{Introdução}

A computação e suas tecnologias estão presentes nos mais diversificados espaços da sociedade e vêm sendo utilizadas para inúmeros fins. Os dispositivos móveis, por exemplo, permitiram que a informação e a comunicação fossem disseminadas, o que possibilitou ao ser humano a capacidade de estar em qualquer espaço físico e ao mesmo tempo conectado com pessoas de outras regiões, fazer a leitura de textos publicados em websites, responder e-mails, averiguar o horário do ônibus, verificar atualizações nas redes sociais, marcar reuniões e até mesmo estudar.

Oferecer acesso para todo tipo de dispositivo é um desafio para os sistemas computacionais, principalmente quando a finalidade do sistema é atender usuários estudantes, uma vez que aprender com mobilidade é uma veracidade. Atualmente, os estudos inerentes à aprendizagem eletrônica discorrem sobre um processo de ensinoaprendizagem baseado no contexto do usuário, que considera o que o estudante está 
fazendo, quem está com ele e onde ele está (WAGNER et al. 2014). O termo estudado é ubiquitous learning (u-learning), que consiste em um paradigma de aprendizagem que é oferecido por Ambientes Virtuais de Aprendizagem que utilizam sensores para perceber as necessidades dos estudantes e por intermédio de atuadores acatam tais exigências, de maneira automática, invisível.

Em conformidade com Hwang et al. (2009), os ambientes educacionais ubíquos surgem da aplicação da computação ubíqua em sistemas computacionais destinados ao ensino e à aprendizagem. Constituem-se de recursos que pretendem satisfazer as necessidades dos estudantes, dado que cada aluno possui seus próprios métodos de aprendizagem, horário para estudar, preferência por formato de material didático, um determinado nível de expertise, local predileto para realizar suas atividades e seu ritmo de estudar. Assim, é importante oferecer ferramentas que procuram atender tais necessidades e que oferecem acesso ao conteúdo educacional independente de local e dispositivo, garantindo que a informação seja acessada no momento propício para o estudante.

$\mathrm{Na}$ literatura há diversas iniciativas, em centros de pesquisa, que buscam explorar a computação ubíqua em ambientes virtuais de ensino e aprendizagem. Cita-se como exemplo o estudo precursor de ubiquitous learning de Ogata e Yano (2004), o qual consistiu na construção de um ambiente educacional destinado ao ensino da língua Japonesa. O mesmo considera a posição geográfica do usuário para personalizar a sua interface gráfica. Outros estudos significativos vêm surgindo nos últimos anos sobre diferentes formas de captura de contexto (informação), para possibilitar a esses ambientes a capacidade de personalização e adequação. Existem investigações que se concentram em informações específicas como estilos de aprendizagem (HSIEH et al. 2011), histórico de interações (LI et al. 2013), localização do usuário (FERREIRA et al. 2015), entre outros, e outras que se especializam em utilizar mais de um tipo de informação do usuário para se personalizar.

No âmbito educacional existe um enumerado campo de estudos que tentam prover novas ferramentas instrucionais para apoiar a aprendizagem. Um objeto de investigação é o uso de tutores virtuais em ambientes computacionais para apoiar o estudante. Cita-se como exemplo as investigações que se concentram em utilizar agentes conversacionais para auxiliar estudantes no aprendizado de conteúdos específicos como o agente Blaze, que contribui com o aprendizado de estudantes de Matemática (AGUIAR et al. 2011), AGEbot, que foi construído para solucionar dúvidas do usuário sobre patologias associadas à epilepsia (FOSSATTI et al. 2011), entre outros. Esses agentes possuem a finalidade de oferecer meios para transmitir informações sobre o conteúdo, mas em muitos casos ignoram as intenções e informações sobre o utilizador.

Nessa perspectiva, esta pesquisa tem a finalidade de apresentar um agente conversacional concebido para propiciar ao estudante um aprendizado baseado em suas propensões. Para tanto, esse agente considera duas informações sobre o estudante, são elas: nível de conhecimento e nível de desempenho. Como estudo de caso, o agente foi concebido para apoiar estudantes de Ciência da Computação, especificamente para discentes da disciplina de Engenharia de Software. Nesse sentido, as bases de conhecimentos do agente foram construídas para contemplar estudantes com diferentes níveis de conhecimento e desempenho na disciplina mencionada. 
$\mathrm{O}$ agente conversacional intitula-se Ubibot e foi construído para ser acoplado junto ao Ambiente Virtual de Aprendizagem Moodle. Salienta-se que se optou por utilizar o Moodle, pois é o sistema educacional mais utilizado por instituições de ensino brasileiras (GALAFASSI et al. 2013), possui licença livre e é utilizado na instituição de ensino em que a presente pesquisa foi realizada. O fato de o Moodle ser utilizado por discentes de Ciência da Computação contribui para esta pesquisa, posto que este estudo também objetiva apresentar alguns testes realizados no âmbito da aplicação do agente em um contexto real, isto é, com estudantes que cursavam a disciplina de Engenharia de Software. Assim, acredita-se que durante as avaliações, os alunos teriam mais facilidade para considerar aspectos negativos e positivos sobre o agente e não sobre o Moodle.

Para apresentar a pesquisa realizada, este artigo está estruturado da seguinte maneira: a segunda seção apresenta uma contextualização sobre os princípios condutores para o estudo, fundamentando os agentes conversacionais, relatando algumas pesquisas já elaboradas no que se refere ao estado da arte; a terceira seção apresenta o Ubibot, descrevendo como ocorre o funcionamento do mesmo; a quarta seção visa relatar os procedimentos utilizados para divulgação do agente e realização de testes; por fim, a quinta seção evidencia as principais contribuições desta pesquisa e elenca sugestões para investigações futuras.

\section{Agentes Conversacionais}

Os agentes conversacionais, também denominados chatterbots, vêm sendo estudados há mais de duas décadas. Existem várias pesquisas que problematizam a construção de tais artefatos e que apresentam resultados satisfatórios, no que se refere à prototipação, construção e utilização dos mesmos. Um contexto investigado é o educacional, na medida em que os mesmos podem ser utilizados para beneficiar os processos de ensino e aprendizagem, atuando como entidades que simulam uma conversa com o estudante, como instrumentos de complementação de aprendizado (KUMAR; ROSÉ, 2011).

Esses recursos possuem como característica principal o comportamento conversacional verbal, que normalmente se assemelha com a de um professororientador. Com isso se estabelece um determinado nível de relacionamento com o usuário, com a intenção de aumentar a satisfação do estudante durante a interação com o Ambiente Virtual de Aprendizagem e apropriação do conteúdo. Desse modo, são capazes de transmitir conselhos automatizados e mensagens informativas para o usuário (BICKMORE et al. 2010), podendo ser relacionadas ao conteúdo de uma disciplina ou não.

Algumas investigações já realizadas serão detalhadas no decorrer desta seção, pois contribuem com o estado da arte e permitem uma compreensão mais detalhada sobre o que vem sendo pesquisado, como quais os procedimentos utilizados e resultados obtidos a partir de tais experimentos. As mesmas constituem-se de publicações que se relacionam com o assunto da presente pesquisa, isto é, estão contextualizadas no âmbito educacional ou de consciência de contexto. Em paralelo com as investigações que serão descritas a seguir, dois artigos imbricados a esta pesquisa foram publicados por Paschoal et al. (2016a) e Paschoal et al. (2016b). Esses textos descrevem contribuições da literatura para a prototipagem do agente Ubibot. 
Moraes et al. (2016) argumentam que os agentes conversacionais podem ser explorados como abordagem metodológica para personalizar disciplinas de computação, estimulando e aumentando o engajamento dos estudantes. No estudo, os autores utilizaram os conceitos inerentes a agentes para apoiar o ensino de Inteligência Artificial, especificamente o conteúdo de agentes reativos. Para isso, propuseram como atividade didática a seus estudantes a construção de um agente que possuísse bases de conhecimentos construídas na linguagem $\mathrm{AIML}^{1}$ (Artificial Intelligence Markup Language) e que englobasse o conteúdo abordado na disciplina. Os resultados da pesquisa demonstraram que a hipótese foi comprovada, isto é, a prática da construção do agente ampliou o empenho dos alunos na disciplina.

Enquanto Moraes et al. (2016) utilizaram a construção do agente como abordagem metodológica para apoiar o ensino de Inteligência Artificial, Bickmore et al. (2010) conduziram um estudo a partir da implementação de um agente, com a hipótese que um agente conversacional poderia auxiliar pacientes enfermos. Sobre educação em saúde, vale considerar que os usuários não adeptos à utilização de computadores, conseguiriam interagir com a interface e julgariam a mesma como natural, isto é, os usuários não encontram dificuldades ao utilizar o recurso computacional. Para tanto, apresentaram o agente a pacientes e obtiveram resultados satisfatórios, concluindo que o agente proveu um ambiente de baixa pressão em que os usuários são livres para questionar tanto quanto necessitarem.

Bickmore et al. (2009) partiram da emblemática que mobilidade e consciência de contexto são direções de pesquisas importantes para tais agentes. Nesse sentido, os autores apresentam um agente construído para dispositivos móveis que incentiva o usuário à prática de atividades físicas, particularmente o agente tenta motivar $\mathrm{o}$ utilizador a caminhar mais. Os diálogos do agente foram construídos em script derivado de XML (eXtensible Markup Language). O agente consegue ficar ciente de contexto por intermédio de um pedômetro. Os autores, por meio de experimentos, comprovaram duas suposições: (i) a consciência de contexto permitiu uma maior ligação social entre humano-computador e (ii) a consciência de contexto fez com que o usuário praticasse mais atividades físicas.

Diferente dos estudos elencados, a presente pesquisa demonstra um agente conversacional que busca satisfazer estudantes de Engenharia de Software. O Ubibot foi construído para apoiar a aprendizagem ubíqua, por isso contém recursos capazes de identificar informações sobre o aprendizado dos estudantes. Além disso, ele pode ser executado em diferentes dispositivos, por intermédio de browsers de smartphones, tablets e desktops. Os trabalhos relacionados apontam diferentes iniciativas, que não têm a finalidade de apoiar o ensino de Engenharia de Software, nem utilizam informações de expertise sobre o conteúdo e de desempenho do aluno na disciplina. Todavia, os estudos correlatados apresentam iniciativas que serviram de motivação para o desenvolvimento desta pesquisa, dado que contém resultados promissores.

Esta seção teve o intuito de apresentar brevemente algumas questões que norteiam o contexto desta pesquisa. Salienta-se que não foram discutidos aspectos sobre questões técnicas de implementação e arquitetura dos chatterbots, dado que nem todas

\footnotetext{
${ }^{1}$ Disponível em: < https://goo.gl/FstMX3>
} 
as pesquisas mencionadas apresentavam características técnicas do agente. Ressalta-se que os chatterbots normalmente são extensões da arquitetura A.L.I.C.E (WALLACE, 2000), posto que tal arquitetura é considerada uma referência na área (SOUZA; MORAES, 2015), assim, as bases de conhecimentos normalmente são implementadas em linguagem de marcação derivada de XML, como AIML, carecendo apenas de uma máquina de inferência para executá-las.

\section{Agente Ubibot}

Esta pesquisa apresenta o Ubibot, um agente conversacional desenvolvido em linguagem de marcação AIML, com uma máquina de inferência denominada Program $\mathrm{O}^{2}$. Integra dois módulos com o intuito de oferecer um grau de ubiquidade para estudantes de Engenharia de Software.

\subsection{Características gerais}

O Ubibot foi construído para ser executado junto ao ambiente Moodle (versão 3.0), como uma extensão, no formato de bloco HTML. Ele possui bases de conhecimentos que contemplam o conteúdo programático da disciplina Engenharia de Software. Também tem bases comuns que abrangem assuntos não relacionados à disciplina, como cumprimentos, gostos populares, perfil, entre outros. Seu conhecimento foi representado em AIML, compondo-se por numerosas tratativas, com o intuito de controlar sentenças repetitivas, o andamento global da conversação, sentenças desconhecidas, entre outros. Nesse sentido, além dos comandos básicos, as seguintes tags popularizam as bases do Ubibot: $<$ random $>,<$ think $>,<$ set $>,<$ get $>,<$ star $>,<$ that $>$, entre outras.

O Ubibot pode ser executado em diferentes interfaces de dispositivos, funcionando em navegadores web, como Mozilla Firefox, Google Chrome, entre outros. Sua interface foi construída para ser executada dentro de um bloco no Moodle e por isso, utiliza o framework Bootstrap ${ }^{3}$ para se adequar ao tamanho da tela do dispositivo. $\mathrm{O}$ agente é apresentado na Figura 1.

Figura 1 - Agente Ubibot

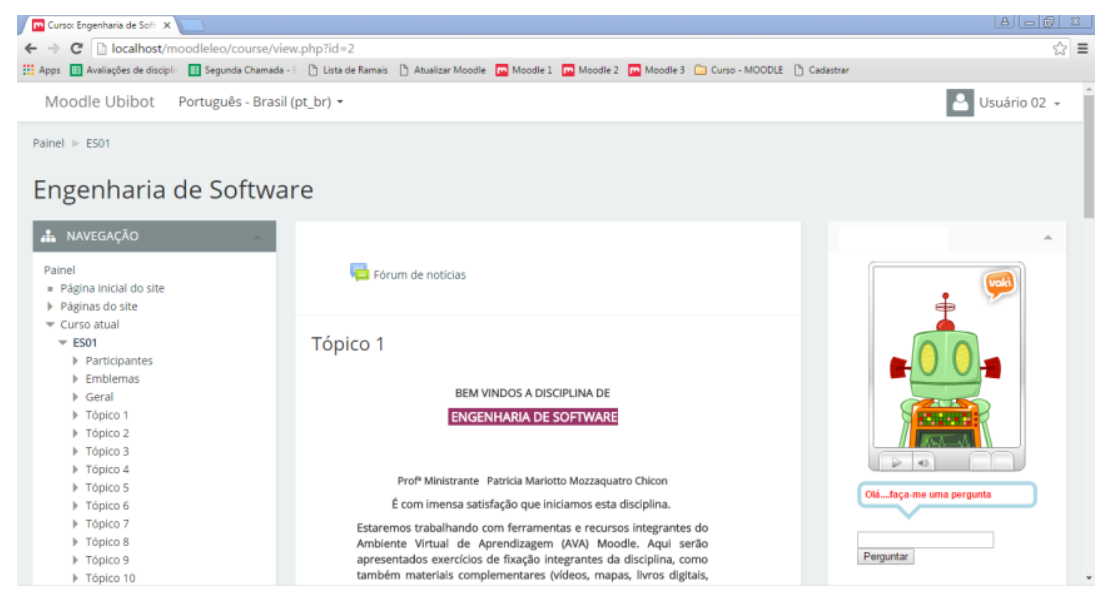

Fonte: Desenvolvido pelos autores

\footnotetext{
${ }^{2}$ Disponível em: $<$ https://goo.gl/ytLskT>

${ }^{3}$ Disponível em: < https://goo.gl/CePXCI >
} 
Conforme descrito anteriormente, a principal característica deste agente consiste na capacidade de adequar suas mensagens de auxílio, em conformidade com o contexto do usuário. Para tanto, foram construídos dois módulos: MINE (Módulo Identificador do Nível de conhecimento prévio de Estudantes) e MIDE (Módulo Identificador do Desempenho de Estudantes), os quais serão detalhados no desenvolvimento desta seção. Esses módulos repercutiram na inserção de duas novas tabelas na base de dados do ambiente Moodle: $m d l \_m i n e$ e $m d l \_m i d e$. Ambas são responsáveis por armazenar dados de contexto (inserções e atualizações) e o $i d$ de cada usuário.

\subsection{Módulos para Identificação de contexto}

O MINE é um módulo que tem a capacidade de identificar o nível de conhecimento do estudante, sobre o assunto abordado na disciplina de Engenharia de Software. Tal módulo é fortemente baseado no padrão SistEx, utilizado no jogo JASPION (KRASSMANN et al. 2015), uma vez que o SistEx é capaz de identificar o nível de conhecimento de estudantes de Redes de Computadores. Assim, o MINE é composto por um questionário com perguntas sobre os assuntos que contemplam o conteúdo de Engenharia de Software.

Esse módulo é acionado quando um estudante matriculado em Engenharia de Software acessa pela primeira vez o Moodle, uma vez que a finalidade dele é averiguar o conhecimento prévio do estudante sobre os conteúdos que serão abordados na disciplina. Logo, o Moodle solicita ao estudante o preenchimento de um questionário para prosseguir e ter acesso à disciplina. Se o aluno respondê-lo, o agente consegue identificar que há uma informação sobre o estudante, disponível na tabela mdl_mine, e se apresentará ao aluno. Caso o aluno não responda, o agente entenderá que o estudante não precisa de sua ajuda e ficará oculto. Assim, em cada acesso realizado, o Ubibot verifica se há contexto identificado, caso não haja, ele solicita ao aluno para que o mesmo preencha o formulário MINE; caso existe, ele será apresentado ao aluno.

O agente classifica o nível de conhecimento do discente em três categorias: básico, intermediário e avançado. Tal informação possibilita entender quais recomendações são mais adequadas para apresentar ao aluno, uma vez que a literatura evidência que um estudante com conhecimento básico pode não compreender mensagens de auxílio mais complexas e um aluno com conhecimento avançado pode ficar frustrado quando recebe conteúdos básicos (POSSOBOM et al. 2014).

Para complementar o MINE, foi construído o MIDE, que é capaz de observar o desempenho do aluno na disciplina. Ele acompanha as notas do discente no Moodle (tabelas $m d l$ grade_grades e $m d l \_g r a d e \_i t e m s$ ) e consegue classificar o desempenho do mesmo: baixo, bom ou excelente. Essa classificação é embasada no estudo realizado por Dias et al. (2009). Salienta-se que o MIDE é acionado sempre que o aluno acessa o ambiente, mas só consegue identificar o nível de desempenho na disciplina quando existem notas disponibilizadas pelo professor. Portanto, enquanto não existem atividades avaliadas na disciplina, ele não consegue nortear o agente. Assim, é evidente a contribuição de cada módulo, um complementa o outro.

Os fatores delimitadores de classificação para cada módulo são descritos em um estudo publicado por Paschoal et al. (2016b). Estes fatores são responsáveis por fazer com que o agente entenda em qual contexto o usuário está. Ressalta-se que o agente só 
considera o nível de conhecimento do estudante enquanto não houver nível de desempenho identificado, pois quando o desempenho é reconhecido ele compreenderá o resultado como um reflexo de alguma situação em que o aluno se encontra, presumindo que o aluno está com dificuldades ou não na disciplina.

As bases de conhecimentos do agente contemplam três tipos de perfis: conhecimento básico e baixo desempenho, conhecimento intermediário e bom desempenho, conhecimento avançado e excelente desempenho. Assim, o Ubibot consegue auxiliar um estudante classificado com baixo desempenho ou com conhecimento básico, por intermédio da mesma base de conhecimento, uma vez que o agente considera cada contexto em momentos diferentes.

\section{Socialização e Testes}

A fim de averiguar o impacto desta proposta, o agente foi socializado com uma turma de quinze estudantes, todos devidamente matriculados na disciplina de Engenharia de Software. Nenhum desses alunos havia cursado essa disciplina anteriormente. A proposta era analisar as percepções dos estudantes quanto aos auxílios realizados pelo agente. Assim, os pesquisadores deste artigo apresentaram o sistema, a proposta da pesquisa e incentivaram os participantes a utilizar o máximo possível o agente para resolver suas dúvidas relacionadas aos conteúdos e atividades da disciplina.

Os participantes utilizaram o agente por um período de cinco semanas. Acreditase que esse período foi suficiente, dado que ele contempla mais de um tópico do conteúdo e é considerável para os alunos se acostumarem com o sistema. Depois desse ínterim, os pesquisadores aplicaram um questionário para aferir se o agente conseguiu desempenhar suas atividades de maneira satisfatória. O questionário aplicado encontrase disponível no seguinte endereço: $<$ https://goo.gl/yZPC5H $>$. Ele contém dezesseis questões, sendo quinze de múltipla escolha que utilizam a escala Likert e uma descritiva. Do total de questões, as principais estão expostas na Figura 2.

Figura 2 - Questões fundamentais

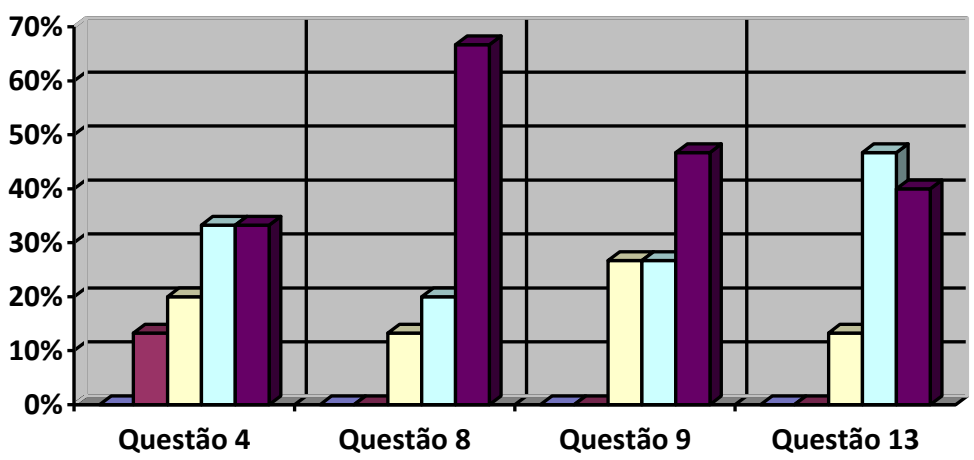

\begin{tabular}{|l|}
\hline Discordo Totalmente \\
$\square$ Discordo \\
$\square$ Indeciso \\
$\square$ Concordo \\
$\square$ Concordo Totalmente
\end{tabular}

Fonte: Desenvolvido pelos autores

Ao serem questionados sobre as sugestões e suporte realizado pelo agente, $33,3 \%$ dos entrevistados concordaram totalmente, evidenciando que o agente ofereceu sugestões claras; $33,3 \%$ concordaram em parte, $20 \%$ destacou que ficou indeciso e $13 \%$ discordaram. É importante enfatizar que os $13,3 \%$ que discordaram em parte, não realizaram todas as atividades existentes na disciplina. Assim, presume-se que os 
mesmos esperavam mais do agente e possivelmente acreditaram que o agente poderia resolver situações adversas. A primeira coluna do gráfico apresenta tais resultados.

A oitava questão buscou descobrir se as informações apresentadas pelo agente são úteis. Por meio das respostas, pôde ser mensurado e demonstrado que a maioria dos avaliadores considerou as informações úteis, uma vez que $66,7 \%$ concordaram totalmente e $20 \%$ concordaram parcialmente. Todavia, $13,3 \%$ discorreram que ficaram indecisos. $\mathrm{O}$ resultado associado à incerteza pode ser alusivo com as bases de conhecimento, pois as mesmas não contemplaram alguns pares conversacionais fora da área, como questões associadas a tempo, dia da semana, etc.

A nona questão teve o objetivo de verificar se os estudantes acreditaram que os auxílios pedagógicos do agente foram realizados conforme os contextos de nível de conhecimento e nível de desempenho. Constatou-se que $46,7 \%$ concordaram totalmente, $26,7 \%$ concordaram em parte. Os resultados constatam que os avaliadores admitiram que as informações foram apresentadas de acordo com o perfil de cada usuário. Ainda, por meio dela, percebe-se que alguns estudantes ficaram indecisos (26,7\%). Essa resposta talvez seja decorrente do fato que os estudantes não eram informados sobre a alteração de contexto e em momento algum sabiam a qual contexto pertenciam. Portanto, é plausível supor que esses estudantes não souberam responder se as respostas eram adequadas ao contexto e tomar uma decisão.

$\mathrm{Na}$ décima terceira questão investigou-se o critério de satisfação relativo tanto ao agente quanto ao Moodle. Como efeito constatou-se que $40 \%$ dos entrevistados ficaram totalmente satisfeitos, $46,7 \%$ declararam que ficaram satisfeitos em parte e 13,3\% expressaram indecisão. A opção de indecisão aparece com um valor importante o que pode significar que alguns estudantes podem ter tido outra expectativa do agente. Essa pontuação pode estar relacionada com a tecnologia utilizada no desenvolvimento do agente, a base de conhecimento ou inclusive ao método como o agente foi utilizado pelo estudante.

No geral, os resultados apresentados evidenciam que o agente obteve uma boa aceitação pelos avaliadores. Tais resultados eram esperados, pois ressaltam a importância de se construir ferramentas para apoiar o ensino dos alunos. É importante frisar que a autoria docente também é fundamental, mas por diversos motivos, alguns docentes não conseguem arranjar tempo para desenvolver tais ferramentas e ainda existem aqueles que não possuem conhecimentos técnicos. Assim, torna-se cada vez mais importante que pesquisadores de áreas relacionadas à Ciência da Computação desenvolvam tais recursos. Ressalta-se que os resultados apresentados nesta seção são fragmentos de uma investigação derivada de um trabalho de conclusão de curso.

\section{Considerações Finais}

Vive-se um período em que os computadores popularizam vigorosamente a atual sociedade. Tais dispositivos estão cada vez mais integrados no dia a dia dos seres humanos. Tamanha integração contribui com o surgimento de iniciativas para fazer com que eles se ajustem às necessidades dos usuários, proporcionando capacidades de identificar informações sobre seus utilizadores e ações benéficas, individualizadas. Desse modo, estudos são realizados para tornar possível a computação ubíqua. Nesse sentido, o âmbito educacional é uma esfera viável de aplicação, uma vez que os 
estudantes do século XXI necessitam de suporte tecnológico para seu aprendizado e pesquisas relacionadas à aprendizagem ubíqua demonstram resultados auspiciosos.

Este artigo teve o objetivo de apresentar um agente conversacional que oferece recomendações com um grau de ubiquidade, visto que o mesmo possui recursos para captura e tratamento de contexto e utiliza tecnologia web responsive para se adaptar em diferentes interfaces. O Ubibot foi socializado com uma turma de graduandos em Ciência da Computação, com a finalidade de ser testado. Os resultados da avaliação revelam que o agente conseguiu satisfazer seus objetivos, contribuindo com a aprendizagem dos estudantes. Todavia, alguns apontamentos dos estudantes revelam que atualizações são necessárias, como a ampliação do número de bases de conhecimento, tratamento de algumas sentenças de interação, entre outras. Além disso, é importante considerar a realização de novas avaliações, trabalhar com uma interface mais amigável e até mesmo com novas formas de detecção de contexto, como os estilos de aprendizagem do aluno e os dados de navegação do usuário.

\section{Referências}

AGUiAR, E. V. B.; TAROUCO, L. M. R.; REATEGUI, E. B. A construção do conhecimento matemático com engajamento e aprimoramento de habilidades cognitivas apoiada por um agente conversacional. Revista Latinoamericana de Tecnología Educativa, v. 10 (2), 2011.

BICKMORE, T. W.; MAUER, D.; BROWN, T. Context awareness in a handheld exercise agent. Pervasive and Mobile Computing, v. 4 (1), p. 226-235, 2009.

BICKMORE, T. W.; PFEIFER, L. M.; BYRON, D.; FORSYTHE, S.; HENAULT, L. E.; JACK, B. W.; SILLIMAN, R.; PAASCHE-ORLOW, M. K. Usability of Conversational Agents by Patients with Inadequate Health Literacy: Evidence from Two Clinical Trials. Journal of Health Communication, v. 15 (2), p. 197-210, 2010.

DIAS, A. F. M.; CERQUEIRA, G. S.; LINS, L. N. Fatores determinantes da retenção estudantil em um curso de graduação em engenharia de produção. Anais do XXXVII Congresso Brasileiro de Educação em Engenharia, 2009.

FERREIRA, L. G. A.; BARBOSA, J. L. V.; GLUZ, J. C.; VICARI, R. UbiGroup: Um Modelo de Recomendação Ubíqua de Conteúdo para Grupos Dinâmicos de Aprendizes. Revista Brasileira de Informática na Educação, v. 23 (3), p. 40-55, 2015.

FOSSATTI, M. C.; RABELLO, R. S.; MARCHI, A. C. B. O uso de um chatterbot em AIML como módulo de interface de um Sistema Tutor Inteligente para auxiliar no aprendizado sobre Epilepsia. Anais do XXII Simpósio Brasileiro de Informática na Educação, SBIE 2011, 2011.

GAlAFASSI, F. P.; GLUZ, J. C.; GALAFASSI, C. Análise Crítica das Pesquisas Recentes sobre as Tecnologias de Objetos de Aprendizagem e Ambientes Virtuais de Aprendizagem. Revista Brasileira de Informática na Educação, v. 21 (3), 2013.

HSIEH, S.; JANG, Y.; HWANG, G.; CHEN, N. Effects of teaching and learning styles on students' reflection levels for ubiquitous learning. Computers \& Education, v. 57, 2011. 
HWANG, G.; YANG. T.; TSAI, C.; YANG, S. J.H. A context-aware ubiquitous learning environment for conducting complex science experiments. Computers \& Education, v. 53 (2), p. 402-413, 2009.

KRASSMANN, A.; PASCHOAL, L. N.; FALCADE, A.; MEDINA, R. JASPION: Jogo Sério Educacional Sensível ao Contexto Integrado ao Ambiente Virtual OpenSim. Anais do XXVI Simpósio Brasileiro de Informática na Educação, SBIE 2015, 2015.

KUMAR, R.; ROSÉ, C. P. Architecture for Building Conversational Agents that Support Collaborative Learning. IEEE Transactions on Learning Technologies, v. 4 (1), p. 21-34, 2011.

LI, M.; OGATA, H.; HOU, B.; UOSAKI, N.; MOURI, K. Context-aware and Personalization Method in Ubiquitous Learning Log System. Educational Technology \& Society, 16 (3), 362-373, 2013.

MORAES, S. M. W.; SANTOS, A. L. L.; REDECKER, M. Prototipação de Chatterbots como Método de Aprendizagem em Cursos de Computação: uma experiência em sala de aula. Anais do XXII Workshop de Informática na Escola, WIE 2016, 2016.

Ogata, H.; Yano, Y. Context-Aware Support for Computer-Supported Ubiquitous Learning. Proceedings of the 2Nd IEEE International Workshop on Wireless and Mobile Technologies in Education, WMTE'04, 2004.

PASCHOAL, L. N.; CHICON, P. M. M.; FALKEMBACH, G. A. M. Ubibot: um Agente Conversacional Ciente do Contexto de Aprendizagem do Usuário. Revista Novas Tecnologias na Educação (RENOTE), v. 14 (1), 2016 a.

PASCHOAL, L. N.; CHICON, P. M. M.; KRASSMANN, A. L.; BINELO, M. O. Ubibot: Agente Inteligente Consciente do Contexto de Aprendizagem do Usuário Integrado ao Ambiente Moodle. Anais do XXI Congreso Internacional de Informática Educativa, TISE, $2016 b$.

POSSOBOM, C. C.; MÜlHBEIER, A. R. K.; NUNES, F. B.; CARVALHO, A.; GOMES, R. B.; MEDINA, R. D. Uma Aplicação Dinâmica para Detectar o Nível de Conhecimento do Aluno. Anais do XXIII Ciclo de Palestras Novas Tecnologias na Educação, CINTED, 2004.

SOUZA, L. S.; MORAES, S. M. W. Construção automática de uma base AIML para Chatbot: um estudo baseado na extração de informações a partir de FAQs. Anais do Encontro Nacional de Inteligência Artificial e Computacional, ENIAC 2015, 2015.

WAGNER, A.; BARBOSA, J, L. V.; BARBOSA, D. N. F. A model for profile management applied to ubiquitous learning environments. Expert System with Applications, v. 41, p. 2023-2034, 2014.

WALLACE, R. S. "The Anatomy of A.L.I.C.E. Artificial Intelligence Foundation", http://www.alicebot.org/anatomy.html. 2000. 